\title{
Aplicación del Value Stream Mapping en el Proceso del Acero en el Sector Construcción
}

Application of the Value Stream Mapping in the Steel Process in the Construction Sector

${ }^{1}$ Gustavo Adolfo Montoya Cárdenas ${ }^{\mathrm{a}}{ }^{2}$ Jorge Rafael Díaz Dumont ${ }^{\mathrm{a}},{ }^{1}$ Leonidas Manuel Bravo Rojas ${ }^{\mathrm{a}}$

\section{RESUMEN}

El presente estudio tiene por finalidad mostrarnos como aplicar el Value Stream Mapping en un caso del sector construcción, en el proceso del acero específicamente. Se debe procesar 2 Ton de acero diariamente, esto quiere decir que se debe cortar, habilitar, colocar y armar el acero que servirán en el colocado de muros y lozas para departamentos de interés social en obras de Lima Metropolitana. Se hacen las estructuras de dos departamentos por día, esta velocidad de producción exige que el tren de actividades se cumpla según lo planificado, para procesar el acero en los tiempos programados, es por esto que el valueStreammapping nos ayuda a planificar los proyectos de mejora de procesos.

Palabras clave: VSM, 5S, SMED, PokaYoke, Kanban, Marcapasos, Lead Time.

\begin{abstract}
The purpose of this study is to show us how to apply the Value Stream Mapping in a case of the construction sector, in the steel process specifically. 2 tons of steel must be processed daily, this means that the steel that will be used in the install action of walls and slabs for social interest departments in works of Metropolitan Lima must be cut, enabled, placed and assembled. The structures of two departments are made per day, this speed of production requires that the train of activities be fulfilled according to the plan, to process shtetel in the programmed times, that is why the values treemapping helps us to plan the projects of process improvement.
\end{abstract}

Keywords: VSM, 5S, SMED, PokaYoke, Kanban, Pacemaker, Lead Time.

\footnotetext{
${ }^{1}$ Universidad Cesar Vallejo

${ }^{2}$ Universidad Nacional Autónoma de Tayacaja Daniel Hernández Morillo

${ }^{a}$ Ingeniero Industrial
} 


\section{INTRODUCCIÓN}

En esta última década, el Perú ha sido una de las economías más dinámicas de América Latina, con una tasa de crecimiento anual promedio de 5,9 por ciento en un entorno de baja inflación en 2,9 por ciento en promedio, (BCRP, 2017). Un contexto externo favorable, políticas macroeconómicas prudentes que fueron implementadas y reformas estructurales en diversos ámbitos convergieron para dar lugar a este escenario de alto crecimiento con baja inflación a pesar de lo acontecido en el panorama internacional (Banco Mundial, 2016).

La actividad de las empresas del sector construcción creció en $2.64 \%$ en el último bimestre del 2017, respecto al mismo periodo de 2016 del mes de enero, figura 1.

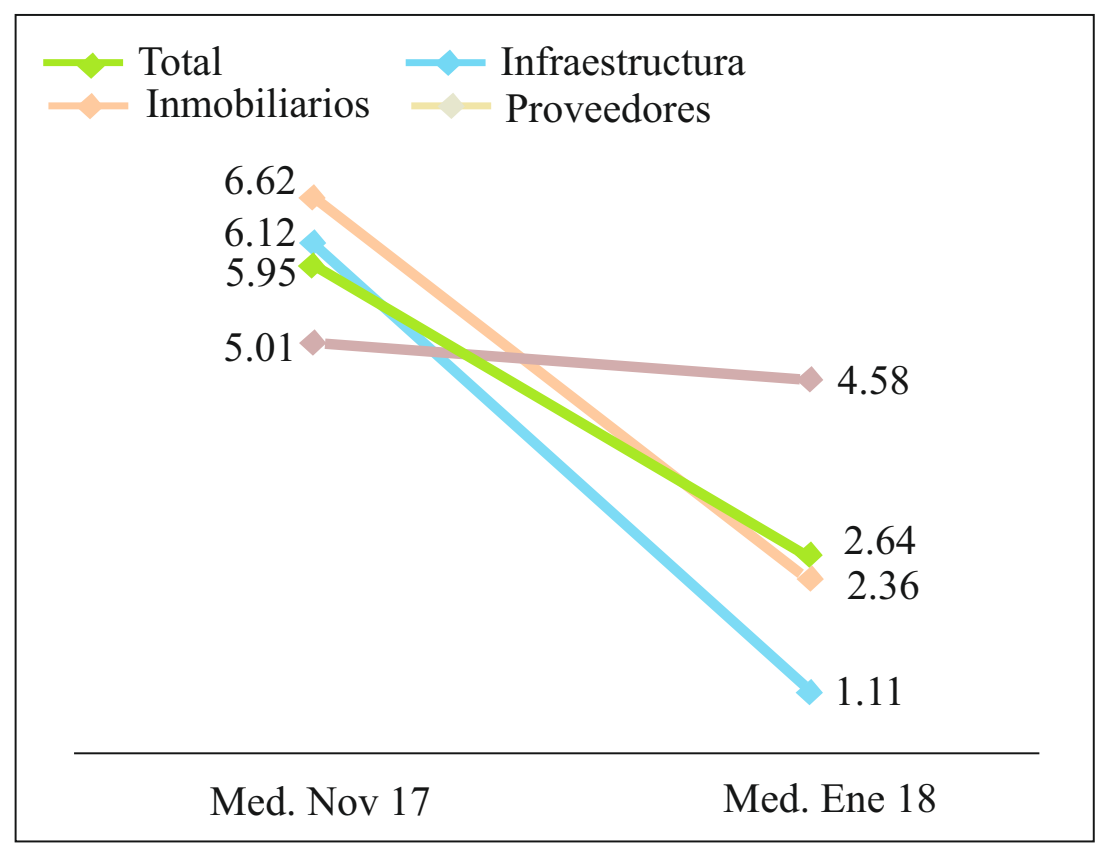

Figura 1. Comparativo entre situación esperada y real sobre la variación en el nivel de las operaciones en las empresas constructoras. (Sexto bimestre 2017 vs. Sexto bimestre 2016 $\%)$

Fuente: Encuesta de Expectativas del IEC CAPECO

El consumo del acero un material recurrente para el desarrollo del sector construcción ha venido creciendo con relación al año base (diciembre 2009), el precio deeste material registra un incremento de $7.42 \%$, siendo elinsumo que, después del ladrillo, registra un menorcrecimiento desde esa fecha.

Los salarios del sector construcción han subido 40.51\%,desde diciembre de 2009, siendo éste el rubro que másse ha incrementado en los últimos cuatro años. 


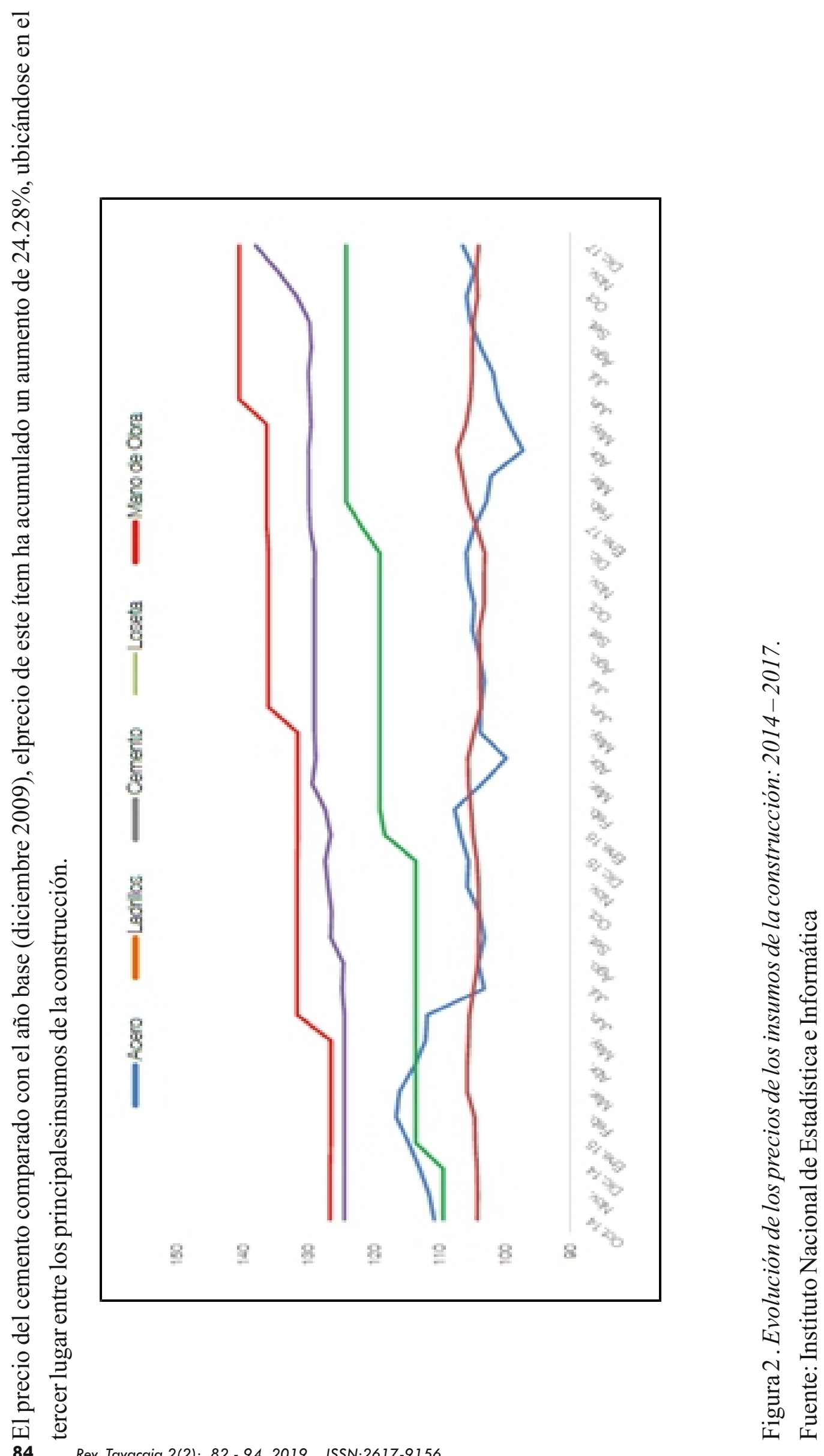


Los distritos de donde se recaba la información son: La Molina, Miraflores, San Borja, San Isidro, Surco, Jesús María, Lince, Magdalena, Pueblo Libre y San Miguel. Donde se sigue desarrollando el sector inmobiliario principalmente. Este desarrollo inmobiliario ha impulsado el incremento del precio por metro cuadrado de estos distritos tal como se aprecia en la figura $\mathrm{N}^{\circ} 3$, los precios se han incrementado desde 600 dólares americanos hasta 1,742 dólares americanos como media de estos distritos.

\section{MATERIALES Y MÉTODOS}

Value Stream Mapping (VSM)

La metodología VSM, basada en el Mapa del Flujo de Materiales e Información de Toyota es una representación gráfica, mediante símbolos específicos, del flujo de materiales y del flujo de información lo largo de la cadena de valor de una familia de productos al conjunto de procesos que contribuyen a transformar la materia prima en producto terminado.

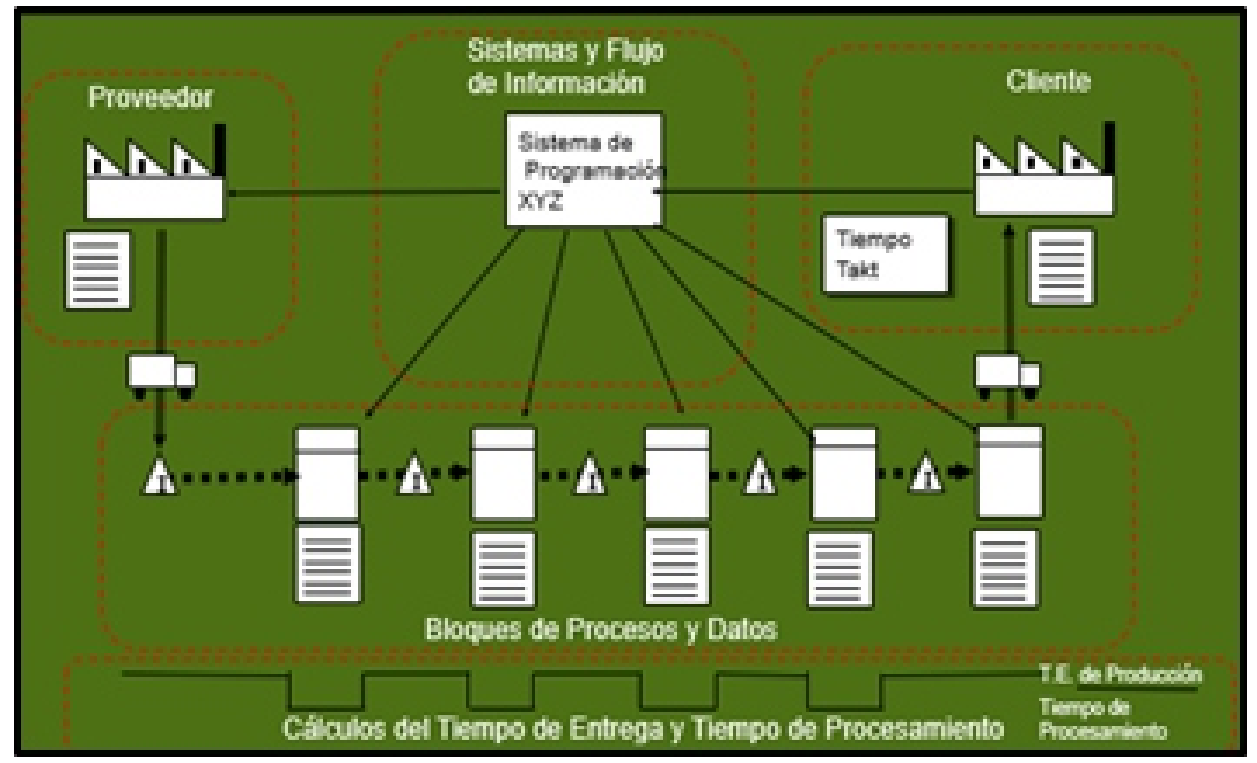

Figura 3. Modelo de Diseño del Mapa de Situación Actual

El VSM, posee una simbología para la comprensión del mapa de valor el cual presenta:

\begin{tabular}{|cl|}
\hline Icono VSM & Representa \\
\hline Recibo & Proceso \\
\hline & Fuentes Externas \\
\hline \hline Cuadro de datos \\
\hline $\begin{array}{c}300 \text { piezas } \\
\text { en 1 dia }\end{array}$ & Inventario \\
\hline \begin{tabular}{|l} 
Usps \\
DIARIO
\end{tabular} & $\begin{array}{l}\text { Envío físico } \\
\text { o Postal }\end{array}$ \\
\hline
\end{tabular}

\begin{tabular}{|cl|}
\hline & $\begin{array}{l}\text { Movimientos } \\
\text { de productos } \\
\text { Terminados al } \\
\text { Cliente }\end{array}$ \\
\hline$\exists$ & $\begin{array}{l}\text { Movimiento del } \\
\text { OBJETO DE VALOR } \\
\text { mediente empuje }\end{array}$ \\
\hline$G$ & Supermercado \\
\hline $\begin{array}{l}\text { Frecuencia máxima } \\
\text { Icantidad }\end{array}$ & $\begin{array}{l}\text { FIFO - Secuencia } \\
\text { Primero en entrar }\end{array}$ \\
\hline FIFO $\rightarrow$ & \begin{tabular}{l} 
Primero en Salir \\
\hline
\end{tabular}
\end{tabular}

\begin{tabular}{|ll|}
\hline & $\begin{array}{l}\text { Kanban de } \\
\text { Transporte }\end{array}$ \\
\hline & $\begin{array}{l}\text { Kanban de } \\
\text { Señal }\end{array}$ \\
\hline$\longrightarrow$ & $\begin{array}{l}\text { "Ver" Programación } \\
\text { de Producción }\end{array}$ \\
\hline$\longrightarrow$ & $\begin{array}{l}\text { Flujo de información } \\
\text { manual }\end{array}$ \\
\hline & $\begin{array}{l}\text { Flujo de información } \\
\text { electrónica }\end{array}$ \\
\hline
\end{tabular}

Figura 4. Iconos VSM (Fuente: See ing the whole-Mapping the Extended Value Stream, 83 


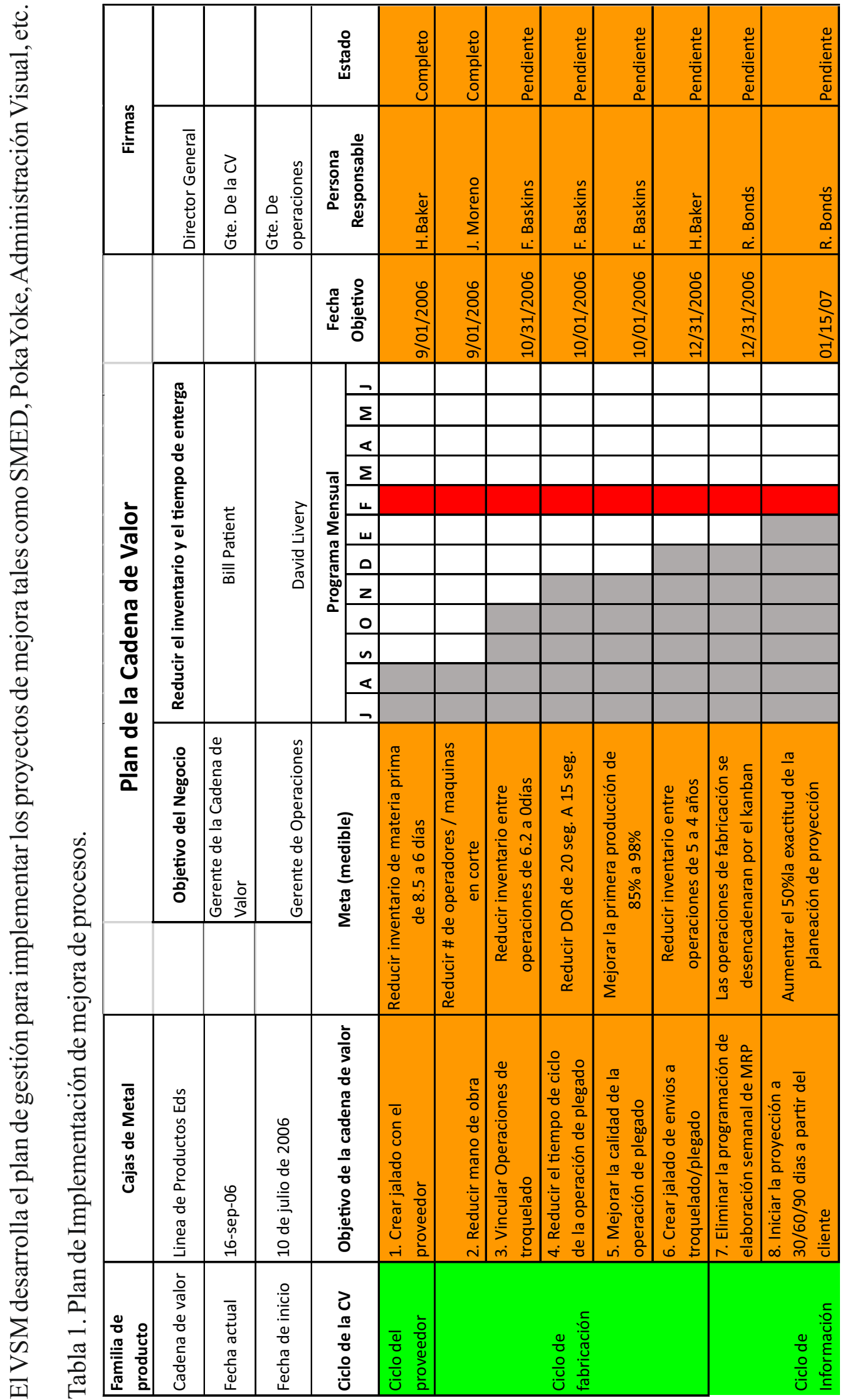

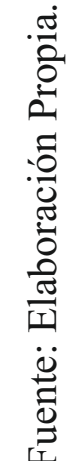




\section{Diagramas de Flujo}

Los diagramas de flujo tienen por finalidad definir el conjunto de actividades que se realizarán en el proceso, podemos definir el inicio y final del proceso representado, también podemos definir los roles que participarán en el proceso. Su simbología esta definida por la ANSI, los cuales son:

Tabla 2. Simbología de diagramas de flujo

\begin{tabular}{|c|c|c|c|}
\hline Simbolo & Representa & Simbolo & Representa \\
\hline & $\begin{array}{l}\text { Simboliza el inicio o } \\
\text { final del diagrama }\end{array}$ & & Conector de padgina \\
\hline & $\begin{array}{l}\text { Indica un punto de } \\
\text { decisibn o alternativa, } \\
\text { en el diagrama }\end{array}$ & & $\begin{array}{l}\text { Conector o enlace con } \\
\text { ef diagrama }\end{array}$ \\
\hline & $\begin{array}{l}\text { Documento, representa } \\
\text { cualquier tipo de } \\
\text { documento que entra at } \\
\text { diagrama }\end{array}$ & & $\begin{array}{l}\text { Actividad; representa } \\
\text { las actividades a } \\
\text { desarrollar }\end{array}$ \\
\hline & $\begin{array}{l}\text { Base de Datos, } \\
\text { representa el banco de } \\
\text { datos de los datos }\end{array}$ & & $\begin{array}{l}\text { Linea de Flujo; } \\
\text { representa la dirección } \\
\text { de la información }\end{array}$ \\
\hline
\end{tabular}

\section{Descripción de la Empresa}

La empresa que será objeto de estudio, se dedica a la construcción de departamentos de interés social, es decir, proyectos de vivienda, que atienden las necesidades de los habitantes con pocas facilidades de acceso a la vivienda propia, esta empresa anualmente entrega en promedio 3,000 departamentosen todos los proyectos desarrollados a nivel nacional, por ello esta empresa se enfoca en brindar departamentos económicos, que cumplen todos los estándares de construcción peruano que es uno de los más exigentes en Latinoamérica, tiene como interés mejorar su proceso constructivo, con la finalidad de reducir costos, el proceso elegido en mejorar el lead time es el proceso del acero, este proceso es un proceso estratégico, debido a que este proceso si no llegar a cumplir con la planificación del día, perjudica todo el tren de actividades de la obra, si el proceso del acero se demora puede perjudicar el proceso de colocado de encofrado, $y$ perjudica el proceso de colocado de concreto.

Tabla 3. Procesos Constructivos

\begin{aligned} & \hline Item Descripción \\ & \hline 01 Limpieza de terreno \\ & 02 Colocar Acero de loza \\ & 03 Instalaciones Sanitarias \\ & 04 Instalaciones eléctricas \\ & 05 Colocar Formaletas \\ & 06 Colocar Concreto \\ & \hline\end{aligned}

La empresa constructora, tiene el proceso del acero que debe ser gestionado de manera eficiente por ser un proceso crítico en su cadena de valor, si este proceso se demora todo el tren de actividades se demorará si acaba adelantándose en su cronograma permite que los demás procesos puedan comenzar con horas de anticipación los procesos subsiguientes. 
Tabla 4. Hoja de Verificación información recogida durante un mes de trabajo.

\begin{tabular}{|c|c|c|c|c|}
\hline Item & Descripción & Defectos & F.R & F.A. \\
\hline $\mathrm{C} 1$ & $\begin{array}{l}\text { Falta un método único de } \\
\text { corte y habilitado }\end{array}$ & 26 & 0.31 & 0.31 \\
\hline $\mathrm{C} 2$ & Falta de planificación de corte & 19 & 0.22 & 0.53 \\
\hline $\mathrm{C} 3$ & $\begin{array}{l}\text { Uso de peones en el proceso } \\
\text { de corte }\end{array}$ & 14 & 0.16 & 0.69 \\
\hline $\mathrm{C} 4$ & $\begin{array}{l}\text { Solo un operario en corte de } \\
\text { acero }\end{array}$ & 11 & 0.13 & 0.82 \\
\hline $\mathrm{C} 5$ & $\begin{array}{l}\text { Herramientas que se } \\
\text { desgastan rápidamente }\end{array}$ & 8 & 0.09 & 0.92 \\
\hline C6 & Inasistencia laboral & 3 & 0.04 & 0.95 \\
\hline $\mathrm{C} 7$ & $\begin{array}{l}\text { Llegan tarde los operarios al } \\
\text { trabajo }\end{array}$ & 2 & 0.02 & 0.98 \\
\hline $\mathrm{C} 8$ & $\begin{array}{l}\text { Prolongados tiempos en } \\
\text { almuerzo }\end{array}$ & 2 & 0.02 & 1.00 \\
\hline
\end{tabular}

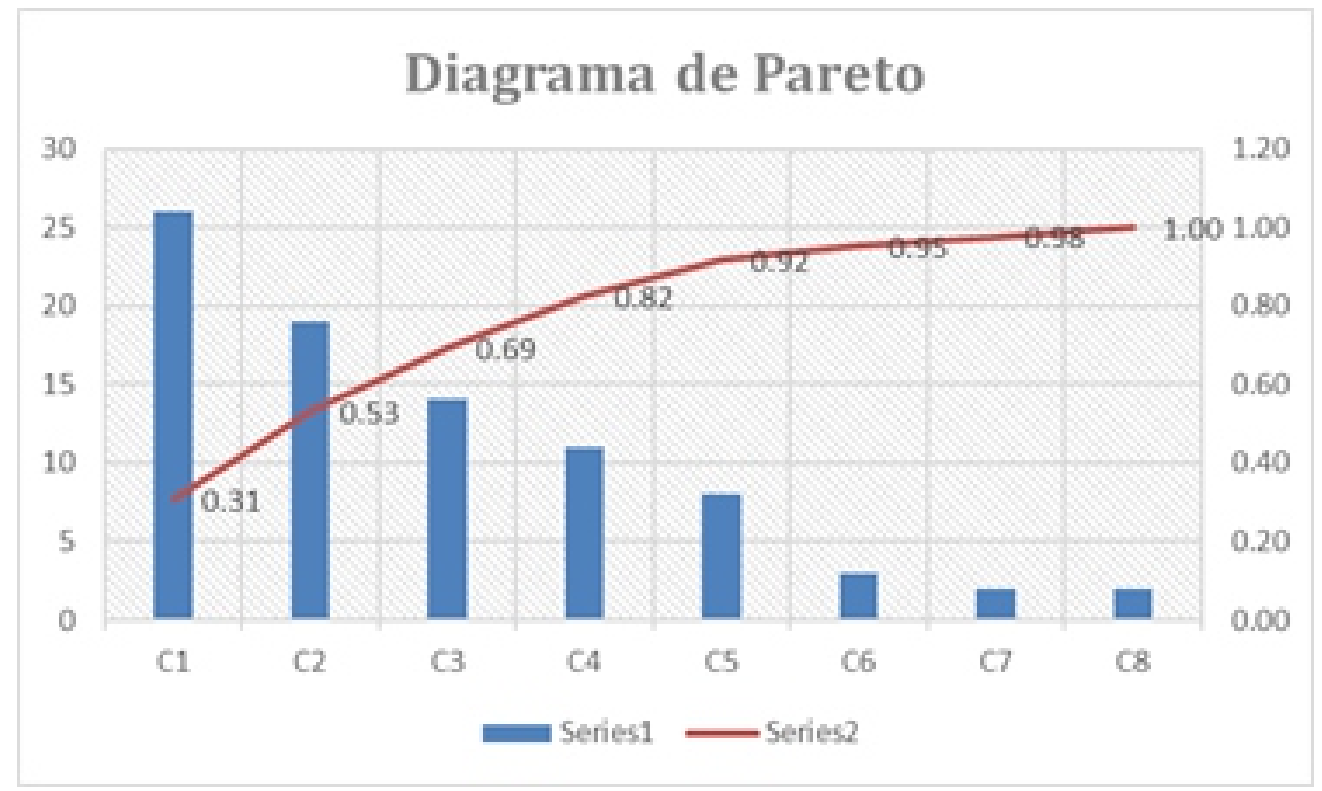

Figura 5. Diagrama de Pareto 
El proceso del Acero tiene un recorrido del almacén general, en ese espacio se almacenan más de 200Tn de acero que servirán para colocarlo en el edificio de la obra, se abastecen en $42 \mathrm{Tn}$ de acero, luego para por el proceso de corte de acero el cual deben ser cortados y clasificado en un trabajo de 2 Tn por día de acero, luego pasan al proceso de habilitado donde serán dobladas según el plan de corte y habilitado esto debe realizarse con precisión pues serán utilizados en el armado de columnas según las clasificaciones de obra, esto quiere decir que en la obra tenemos 6 tipos de columnas que se arman a lo largo de la obra. Seguidamente se ensamblan las columnas según clasificación para luego sea trasladado al piso de la obra que le corresponde debemos mencionar que el proyecto tiene 12 pisos de edificio. Finalmente se coloca la columna o muro según especificación técnica.

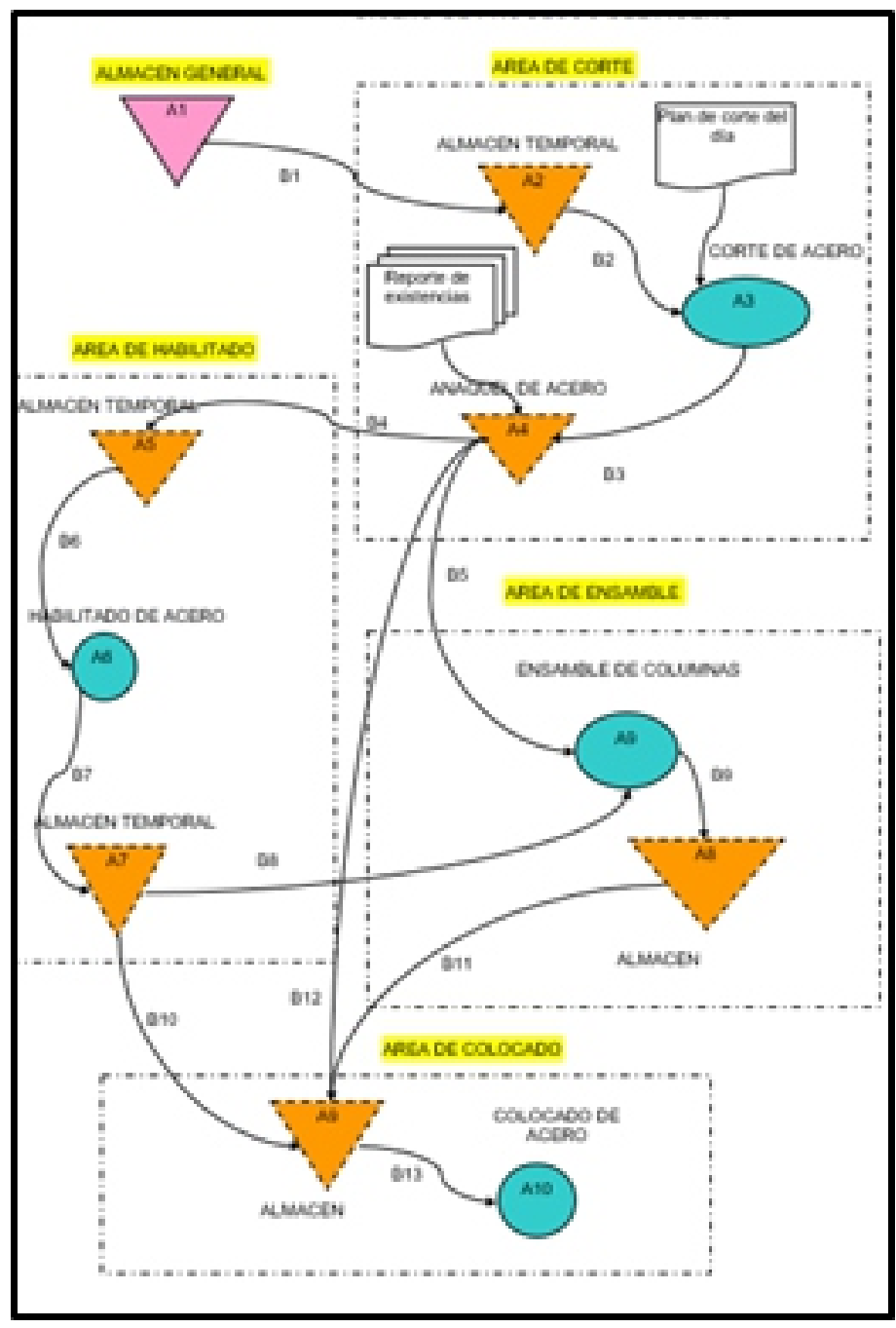

RESUMEN

ALMACEN GENERAL

ALMACÉN TEMPORAL

OPERACIONES

TRANSPORTE

Figura 6. Proceso del Acero 


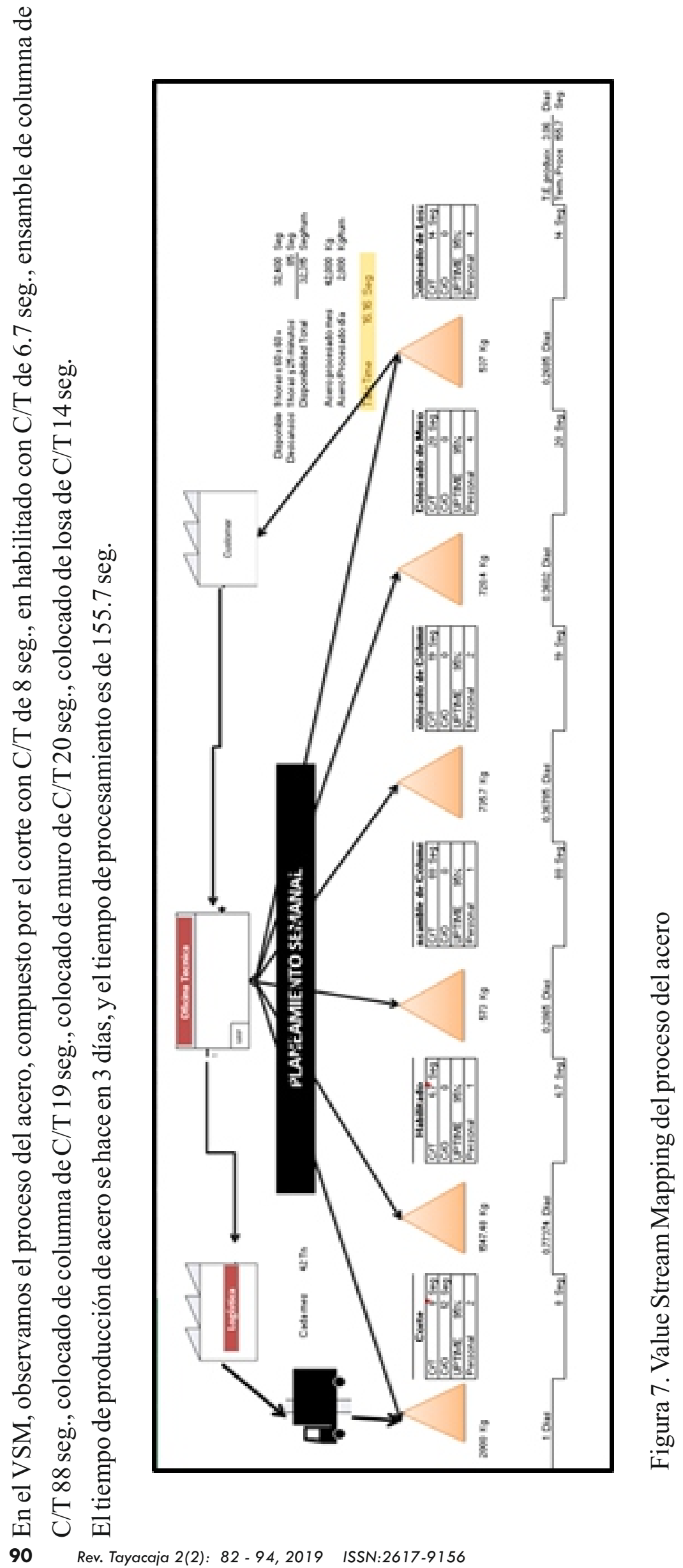


El takt Time de este proceso es 16.16seg., esto nos explica que el ensamble, colocado de columna, colocado de muro y colocado de losa, superan el takt time, de este proceso, por ello estos procesos son candidatos a ser la mejora de los procesos, así mismo, se calcula el número de trabajadores que deben laborar en este proceso esto se calcula así.

Se tiene en la cuadrilla 12 trabajadores lo cual contrasta con el cálculo del número de trabajadores hallado. Número de Trabajadores propuesto.

$$
\begin{gathered}
\# \text { de trabajadores }=\frac{\text { tiempo de procesamiento }}{\text { takt time }} \\
\frac{155.7 \mathrm{seg}}{16.16 \mathrm{seg}}=9.6=>10 \text { trabajadores }
\end{gathered}
$$

Debido a este cálculo de los 12 trabajadores debe reducirse a 10 trabajadores.

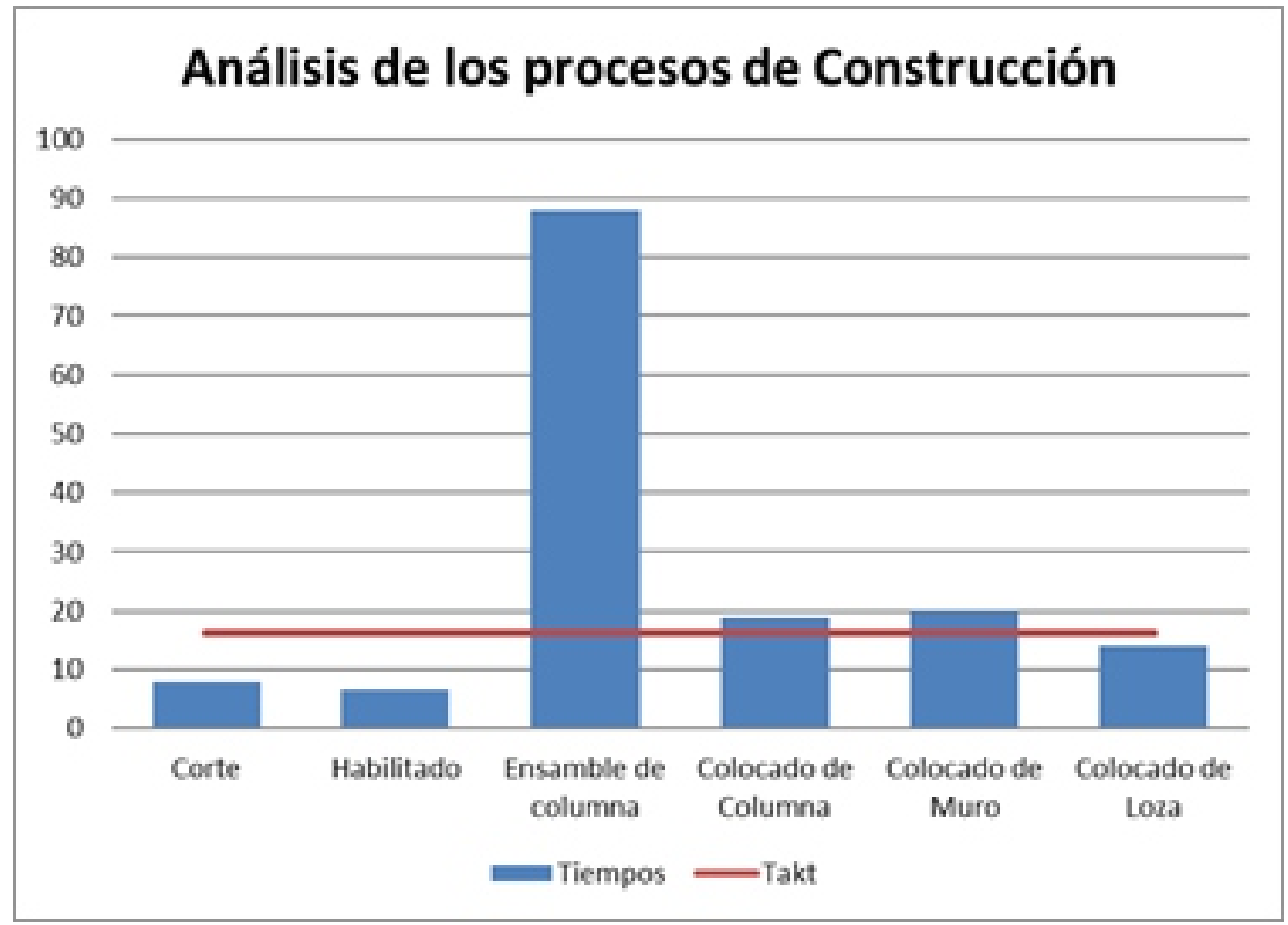

Figura 8. Distribución del Trabajo de los trabajadores

La figura 8. nos explica que el proceso de corte, habilitado y colocado de loza, tienen tiempo de sobra y mas bien esos trabajadores deberían en sus momentos de poca labor deberían apoyar los procesos de Ensamble de columna, colocado de columna, y colocado de muro, pues tienen exceso de trabajo, bajo este calculo no se necesitan mas trabajadores si no más bien saber redistribuir el trabajo asignado y manejo de los tiempos muertos de los procesos.

\section{IMPLEMENTACIÓN DE MEJORAS}

Con este análisis preliminar se identifican varias oportunidades de mejora con relación al takt time $16.16 \mathrm{seg}, \mathrm{y}$ los $\mathrm{C} / \mathrm{T}$ de los procesos sean identificado 3 procesos con 
oportunidad de mejorar y redistribuir los tiempos de trabajos de los 10 trabajadores Para ello los trabajadores de corte de acero, habilitado de acero y colocado de loza, tienen tiempo disponible para poder colaborar con los trabajadores de los procesos de ensamble de acero, colocado de columna, colocado de muro, tienen mas trabajo del que pueden procesar en sus tiempos de trabajo. Por ello se deben trabajar con el Kanban para asignar la carga de trabajo.

Debido a que el tiempo de cambio C/O, es cercano a cero, no necesitan aplicar SMED, pues es la configuración es manual, y no mecánica, se podría automatizar el proceso con tecnología ligada al tratamiento del acero, o podría trabajarse con acero dimensionado, el acero dimensionado es una alternativa para la trabajabilidad del acero teniendo todas las piezas pre dobladas, y cortadas solo utilizado para el ensamblaje y colocado de acero.

Se debe considerar mejorar la gestión de la información, es decir, mejorar el plan de producción del acero, para poder programar las fechas de entregas del acero y sepan cuando deben cumplir con las fechas de entregas y ordenar las entregas de las piezas de acero. Programar con 30/60/90 días el plan de producción del acero, y así poder programar sus entregas. 


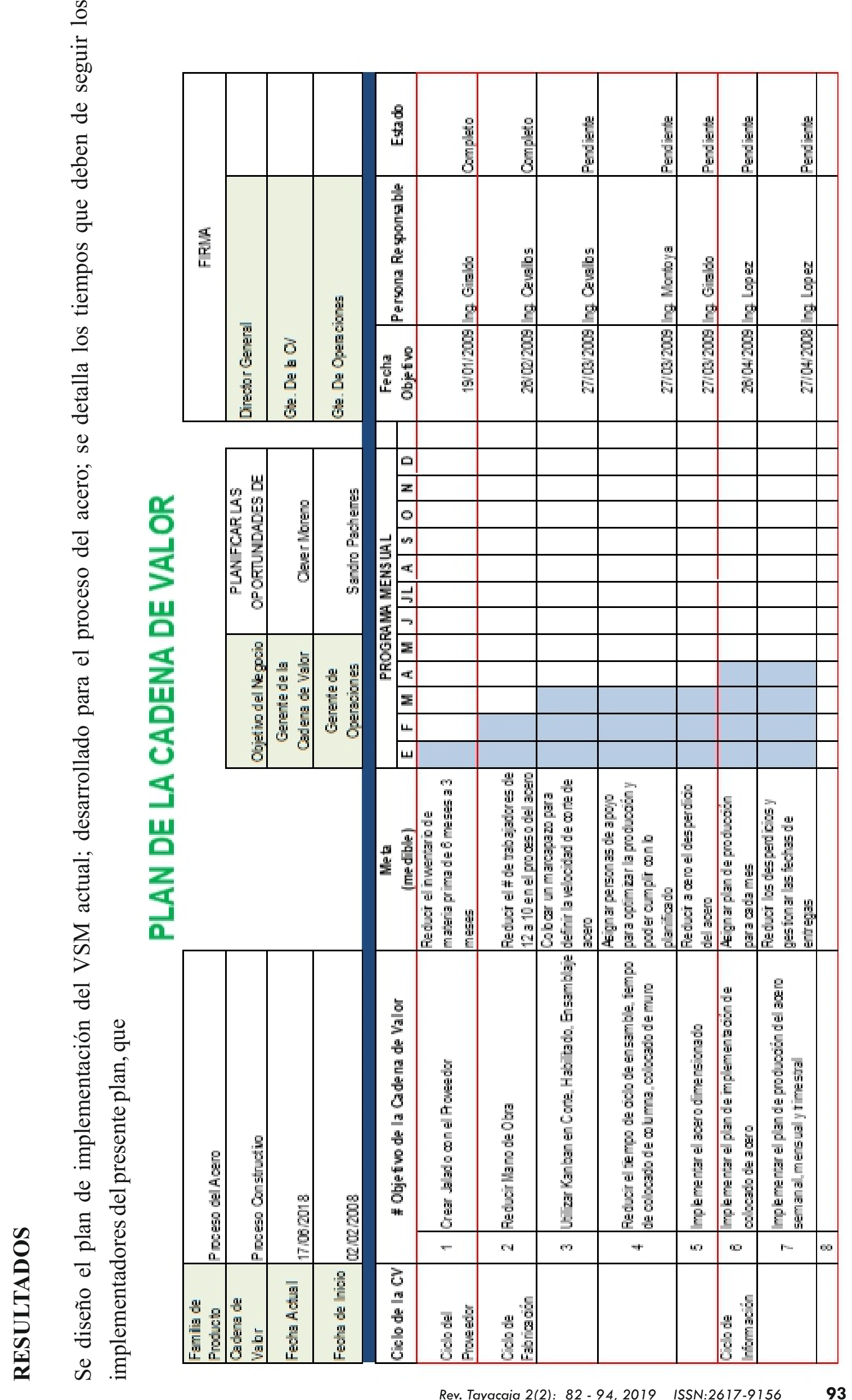




\section{CONCLUSIONES}

El plan de la cadena de valor nos permite preparar los cambios que vamos a realizar en la cadena de valor del acero, y con ello contribuimos a reducir el lead time del proceso del acero, así mismo nos ayuda a definir que herramientas lean utilizaremos para reducir los tiempos, en realidad, las herramientas que utilizaremos será el Lean Construction, por ser la herramienta mas precisa para ajustar los tiempos de entrega y reducir el lead time, es decir tener mayor control con el tren de actividades que le siguen el desarrollo de este proceso, pues el problema central es la falta de cumplimiento de los planes definidos en el proceso constructivo, y desarrollar planes como el Look ahead, las curvas $\mathrm{S}$, son las herramientas que nos ayudarán a complementar este trabajo inicial en la mejora de este proceso.

Gestionar el recurso humano es clave para reducir los costos en este proceso, así mismo, planes de control nos ayuda al cumplimiento de los tiempos, y con ello gestionamos la calidad del proceso.

El uso de herramientas de estudio del trabajo, gestión por procesos, $\mathrm{y}$ herramientas lean no están en conflicto, pues la idea es solucionar los problemas en producción.

\section{REFERENCIAS BIBLIOGRÁFICA}

Womack J., Jones D, Shook J., \& Ferro J., (2009) CreatingLevelPull. USA. 112

Jones D., Womack J., (2003) SeeingThe Whole - Mappingthe Extended ValueStream. USA. 15-17.

Rother M., Shook J., (1999). Learningtosee - valuestreamma ppingto createvalue and eliminate muda. USA 52, 56-68, 78-81.

Liker J., (2004) Las claves del éxito de Toyota 14 principios de gestión del fabricante más grande del mundo. Barcelona. 146-157.

\section{CORRESPONDENCIA}

Mag. Gustavo Montoya Cárdenas gustavo.montoya@productiva.pe 